\title{
When the assignment map is completely positive
}

\author{
Iman Sargolzahi* \\ Department of Physics, University of Neyshabur, Neyshabur, Iran \\ Sayyed Yahya Mirafzali ${ }^{\dagger}$ \\ Department of Physics, Faculty of Science, Vali-e-Asr University of Rafsanjan, Rafsanjan, Iran
}

\begin{abstract}
Finding the general set of system-environment states $\left\{\rho_{S E}\right\}$ for which the reduced dynamics of the system is completely positive (CP) is the subject of some recent works. An advance in this context appeared in [X.-M. Lu, Phys. Rev. A 93, 042332 (2016)], where the problem was solved for the case of $\mathrm{CP}$ assignment map. Here, we restate this result using the framework introduced in [J. M. Dominy et al., Quantum Inf. Process. 15, 465 (2016)]. This, we think, clarifies the mentioned result better and so leads to a generalization of it, straightforwardly.
\end{abstract}

\section{INTRODUCTION}

Consider a closed finite-dimensional quantum system which evolves according to

$$
\rho \rightarrow \rho^{\prime}=A d_{U}(\rho) \equiv U \rho U^{\dagger},
$$

where $\rho$ and $\rho^{\prime}$ are the initial and final states (density operators) of the system, respectively, and $U$ is a unitary operator $\left(U U^{\dagger}=U^{\dagger} U=I\right.$, where $I$ is the identity operator).

In general, the system is not closed and interacts with its environment. We can consider the entire systemenvironment as a closed quantum system which evolves as Eq. (1). So the reduced state of the system after the evolution is given by

$$
\rho_{S}^{\prime}=\operatorname{Tr}_{\mathrm{E}} \circ A d_{U}\left(\rho_{S E}\right)=\operatorname{Tr}_{\mathrm{E}}\left(U \rho_{S E} U^{\dagger}\right)
$$

where $\rho_{S E}$ is the initial state of the combined systemenvironment quantum system and $U$ acts on the whole Hilbert space of system-environment. Now a natural question is what is the relation between the initial state of the system $\rho_{S}=\operatorname{Tr}_{\mathrm{E}}\left(\rho_{S E}\right)$ and its final state $\rho_{S}^{\prime}$ ? Can this relation be represented by a map and - if so - what kind of map?

Consider the case that the set of possible initial states of system-environment is factorized: $\mathcal{S}=\left\{\rho_{S E}=\rho_{S} \otimes\right.$ $\left.\tilde{\omega}_{E}\right\}$, where $\rho_{S}$ are arbitrary states of the system, but $\tilde{\omega}_{E}$ is a fixed state of environment. It is famous that for this special case the reduced dynamics of the system is given by a completely positive (CP) map [1]. But, as we will see in the next section, it is not so in general. Therefore, finding the general set of initial $\rho_{S E}$ for which the reduced dynamics of the system is $\mathrm{CP}$, has become the subject of some recent studies [2-7], which we will review them in Sec. III.

In this context, the most general set of initial $\rho_{S E}$ known prior to our present work (that leads to CP reduced dynamics) has been introduced in Ref. [6]. In fact,

\footnotetext{
* sargolzahi@neyshabur.ac.ir; sargolzahi@gmail.com

† y.mirafzali@vru.ac.ir
}

as proven in Ref. [7], this is the final possible generalization, if we restrict ourselves to the case of CP assignment map.

In this paper, we will restate the result of Ref. [7], using the framework introduced in Ref. [8]. This will help to clarify the result of Ref. [7] better. Specially, our treatment will highlight the condition of $U$-consistency, for arbitrary $U$, which is needed to achieve the result of Ref. [7]. We will give the details in Sec. IV.

In Sec. V, we will generalize the result of Refs. [6, 7]; i.e. we will find a more general set of initial $\rho_{S E}$ which leads to $\mathrm{CP}$ reduced dynamics. So, it includes all the previous results in this context. However, this generalization is rather straightforward, using the framework of Ref. [8].

We will end this paper in Sec. VI, with a summary of our results.

\section{REDUCED DYNAMICS OF OPEN QUANTUM SYSTEM}

There was a tendency to assume the CP maps as the only possible quantum dynamics of a system. But, using Eq. (2), it can be shown simply that it is not so for open quantum systems. In fact, the evolution $\rho_{S} \rightarrow \rho_{S}^{\prime}$ may not be represented by a map, in general [9]. This can be illustrated by the following simple example [8]. Assume that the initial state of the system-environment can be chosen from the set $\mathcal{S}=\left\{\rho_{S}^{(1)} \otimes \rho_{E}^{(1)}, \rho_{S}^{(1)} \otimes \rho_{E}^{(2)}, \cdots\right\}$ and the evolution of the system-environment is given by the swap operator $U_{s w}|\psi\rangle|\phi\rangle=|\phi\rangle|\psi\rangle$. Now for the case that the initial state of the system is $\rho_{S}^{(1)}$, there are (at least) two possible final states:

$$
\begin{aligned}
& \rho_{S}^{(1) \prime}=\operatorname{Tr}_{\mathrm{E}}\left(U_{s w} \rho_{S}^{(1)} \otimes \rho_{E}^{(1)} U_{s w}^{\dagger}\right)=\rho_{E}^{(1)}, \\
& \rho_{S}^{(2) \prime}=\operatorname{Tr}_{\mathrm{E}}\left(U_{s w} \rho_{S}^{(1)} \otimes \rho_{E}^{(2)} U_{s w}^{\dagger}\right)=\rho_{E}^{(2)} .
\end{aligned}
$$

So the evolution from $\rho_{S}$ to $\rho_{S}^{\prime}$ cannot be represented by a map (a map, by definition, assigns to each initial state, e.g. $\rho_{S}^{(1)}$, only one final state). 
Even if the evolution from $\rho_{S}$ to $\rho_{S}^{\prime}$ can be represented by a map, this map is not linear, in general [10]. This also can be represented by the following simple example [11]. Consider an arbitrary non-linear map $\rho_{S} \rightarrow \Phi\left(\rho_{S}\right)$. Assume that the set of passible initial $\rho_{S E}$ is $\mathcal{S}=\left\{\rho_{S} \otimes \Phi\left(\rho_{S}\right)\right\}$ and the evolution of the system and environment is again given by the swap operator. So $\operatorname{Tr}_{\mathrm{E}}\left(U_{s w} \rho_{S} \otimes \Phi\left(\rho_{S}\right) U_{s w}^{\dagger}\right)=\Phi\left(\rho_{S}\right)$, which is, by assumption, a non-linear function of the initial state $\rho_{S}$. Therefore, in order that Eq. (2) leads to a linear map from $\rho_{S}$ to $\rho_{S}^{\prime}$, there must be some restrictions on the set of possible initial states $\rho_{S E}$ or on the possible evolution $U[8,12]$.

However, if the reduced dynamics of the system from $\rho_{S}$ to $\rho_{S}^{\prime}$ can be given by a linear map $\Psi$, then it can be shown readily that this $\Psi$ is Hermitian, i.e. maps each Hermitian operator to a Hermitian operator (details are given in Sec. IV). Now, an important result is that for each linear trace-preserving Hermitian map from $\rho_{S}$ to $\rho_{S}^{\prime}$, there exists an operator sum representation in the following form:

$$
\rho_{S}^{\prime}=\sum_{i} e_{i} \tilde{E}_{i} \rho_{S} \tilde{E}_{i}^{\dagger}, \quad \sum_{i} e_{i} \tilde{E}_{i}^{\dagger} \tilde{E}_{i}=I_{S},
$$

where $\tilde{E}_{i}$ are linear operators and $e_{i}$ are real coefficients $[8,13,14]$. Note that the relation $\sum_{i} e_{i} \tilde{E}_{i}^{\dagger} \tilde{E}_{i}=I_{S}$ comes from the fact that the map is trace-preserving, as expected from Eq. (2). It is also worth noting that $\tilde{E}_{i}$ and $e_{i}$ are fixed, i.e. they are independent of initial state $\rho_{S}$.

If all of the coefficients $e_{i}$ in Eq. (3) are positive, then we call the map completely positive and rewrite Eq. (3) in the following form:

$$
\rho_{S}^{\prime}=\sum_{i} E_{i} \rho_{S} E_{i}^{\dagger}, \quad \sum_{i} E_{i}^{\dagger} E_{i}=I_{S},
$$

where $E_{i} \equiv \sqrt{e_{i}} \tilde{E}_{i}$. In the languge of Ref. [8], we should call a map which is given by Eq. (4), a completely positively trace-preserving extensible map. However, in this paper, we simply refer to such a map a completely positive $(\mathrm{CP})$ map.

The simplest standard example which leads to CP reducd dynamics is when the initial state of the systemenvironment is $\rho_{S E}=\rho_{S} \otimes \tilde{\omega}_{E}$, where $\rho_{S}$ is an arbitrary state of the system, but $\tilde{\omega}_{E}$ is a fixed state of environment. For this case, it can be shown simply that Eq. (2), for an arbitrary $U$, gives us a CP map [1]

Besides the factorized initial states $\rho_{S} \otimes \tilde{\omega}_{E}$, there exist other sets of initial states $\rho_{S E}$ for which the reduced dynamics is CP for arbitrary $U$. This is the subject of some recent works $[2-7]$, which we will review them in the next section.

\section{SETS OF INITIAL STATES WHICH LEAD TO CP REDUCED DYNAMICS}

As we have seen in the previous section, the reduced dynamics of an open quantum system is not given by a
$\mathrm{CP}$ map, in general. In fact, the CP-ness of the reduced dynamics has been proven only for some restricted sets of initial $\rho_{S E}$, which we will review them in the following.

The simplest case which leads to the $\mathrm{CP}$ reduced dynamics is when the initial state of the systemenvironment is factorized, i.e. when the set of possible initial $\rho_{S E}$ is

$$
\mathcal{S}=\left\{\rho_{S E}=\rho_{S} \otimes \tilde{\omega}_{E}\right\},
$$

where $\rho_{S}$ are arbitrary states of the system, but $\tilde{\omega}_{E}$ is a fixed state of environment. Let the eigen-decomposition of $\tilde{\omega}_{E}$ be as $\tilde{\omega}_{E}=\sum_{l} \lambda_{l}\left|\mu_{E}^{(l)}\right\rangle\left\langle\mu_{E}^{(l)}\right|\left(\lambda_{l} \geq 0, \sum_{l} \lambda_{l}=1\right)$. So, for an arbitrary $U$ in Eq. (2), we have

$$
\begin{gathered}
\rho_{S}^{\prime}=\operatorname{Tr}_{E}\left(U \rho_{S} \otimes \tilde{\omega}_{E} U^{\dagger}\right) \\
=\sum_{k l} \lambda_{l}\left\langle k_{E}|U| \mu_{E}^{(l)}\right\rangle \rho_{S}\left\langle\mu_{E}^{(l)}\left|U^{\dagger}\right| k_{E}\right\rangle \\
=\sum_{k l} E_{k l} \rho_{S} E_{k l}^{\dagger}, \quad \sum_{k l} E_{k l}^{\dagger} E_{k l}=I_{S},
\end{gathered}
$$

where $\left\{\left|k_{E}\right\rangle\right\}$ is an orthonormal basis of the Hilbert space of the environment $\mathcal{H}_{E}$ and $E_{k l} \equiv \sqrt{\lambda_{l}}\left\langle k_{E}|U| \mu_{E}^{(l)}\right\rangle$ are linear operators on the Hilbert space of the system $\mathcal{H}_{S}$. In addition, $I_{S}$ is the identity operator on $\mathcal{H}_{S}$. Therefore, the dynamics of the factorized initial $\rho_{S E}$ always reduces to a CP map.

In addition to the factorized initial states, one can find other possible initial sets which also yield $\mathrm{CP}$ reduced dynamics. The first, introduced in Ref. [2], is

$$
\mathcal{S}=\left\{\rho_{S E}=\sum_{i} p_{i}\left|\tilde{i}_{S}\right\rangle\left\langle\tilde{i}_{S}\right| \otimes \tilde{\omega}_{i}\right\}
$$

where $\left\{p_{i}\right\}$ is arbitrary probability distribution $\left(p_{i} \geq 0\right.$, $\left.\sum_{i} p_{i}=1\right)$, but $\left\{\left|\tilde{i}_{S}\right\rangle\right\}$ is a fixed orthonormal basis for $\mathcal{H}_{S}$ and $\tilde{\omega}_{i}$ are fixed density operators on $\mathcal{H}_{E}$. Let $\tilde{\Pi}_{i}=$ $\left|\tilde{i}_{S}\right\rangle\left\langle\tilde{i}_{S}\right|$ and the eigen-decomposition of $\tilde{\omega}_{i}$ be as $\tilde{\omega}_{i}=$ $\sum_{l} \lambda_{i l}\left|\mu_{E}^{(i l)}\right\rangle\left\langle\mu_{E}^{(i l)}\right|$. Now, for arbitrary $U$ in Eq. (2), we have

$$
\begin{gathered}
\rho_{S}^{\prime}=\operatorname{Tr}_{E}\left(U\left(\sum_{i} p_{i} \tilde{\Pi}_{i} \otimes \tilde{\omega}_{i}\right) U^{\dagger}\right) \\
=\sum_{i k l} p_{i} \lambda_{i l}\left\langle k_{E}|U| \mu_{E}^{(i l)}\right\rangle \tilde{\Pi}_{i}\left\langle\mu_{E}^{(i l)}\left|U^{\dagger}\right| k_{E}\right\rangle \\
=\sum_{i k l} \lambda_{i l}\left\langle k_{E}|U| \mu_{E}^{(i l)}\right\rangle \tilde{\Pi}_{i} \rho_{S} \tilde{\Pi}_{i}\left\langle\mu_{E}^{(i l)}\left|U^{\dagger}\right| k_{E}\right\rangle \\
=\sum_{i k l} E_{i k l} \rho_{S} E_{i k l}^{\dagger}, \quad \sum_{j} E_{i k l}^{\dagger} E_{i k l}=I_{S},
\end{gathered}
$$

where $E_{i k l} \equiv D_{i k l} \tilde{\Pi}_{i}$ and $D_{i k l} \equiv \sqrt{\lambda_{i l}}\left\langle k_{E}|U| \mu_{E}^{(i l)}\right\rangle$. As we see, Eq. (8) is in the form of Eq. (4); so it is a CP map.

It is also worth noting that, in addition to the above CP map in Eq. (8), one can find other CP maps which equivalently describe the reduced dynamics of the system. This is due to the fact that one can find more than 
one CP assignment maps $\Lambda$ such that their effects on all $\rho_{S} \in \operatorname{Tr}_{\mathrm{E}} \mathcal{S}$ are the same [15]. (The assignment map is a map which assigns to each $\rho_{S} \in \operatorname{Tr}_{E} \mathcal{S}$ a $\rho_{S E} \in \mathcal{S}$ such that $\operatorname{Tr}_{\mathrm{E}}\left(\rho_{S E}\right)=\rho_{S}$.) Obviously, the effects of these different assignment maps $\Lambda$ on (some of the) states $\rho_{S} \notin \operatorname{Tr}_{\mathrm{E}} \mathcal{S}$ are different.

The result of Ref. [2] is then extended in Refs. [3, 4] where it has been shown that the set of initial states

$$
\begin{gathered}
\mathcal{S}=\left\{\rho_{S E}=\bigoplus_{i=1}^{m} p_{i} \rho_{S}^{(i)} \otimes \tilde{\omega}_{i}\right\}, \\
\mathcal{H}_{S}=\mathcal{H}_{S}^{(1)} \oplus \mathcal{H}_{S}^{(2)} \oplus \cdots \oplus \mathcal{H}_{S}^{(m)},
\end{gathered}
$$

also yields CP reduced dynamics, for arbitrary $U$ in Eq. (2). In Eq. (9), $\left\{p_{i}\right\}$ is arbitrary probability distribution, $\rho_{S}^{(i)}$ is an arbitrary state on $\mathcal{H}_{S}^{(i)}$, but $\tilde{\omega}_{i}$ are fixed states on $\mathcal{H}_{E}$.

The next generalization was given in Refs. $[4,5]$, where the $\mathrm{CP}$ reduced dynamics was proven for the following initial states $\rho_{S E}$ and arbitrary $U$ in Eq. (2):

$$
\begin{gathered}
\mathcal{S}=\left\{\rho_{S E}=\bigoplus_{i=1}^{m^{\prime}} p_{i} \tilde{\omega}_{S E}^{(i)}+\bigoplus_{i=m^{\prime}+1}^{m} p_{i} \rho_{S}^{(i)} \otimes \tilde{\omega}_{i}\right\}, \\
\mathcal{H}_{S}=\mathcal{H}_{S}^{(1)} \oplus \mathcal{H}_{S}^{(2)} \oplus \cdots \oplus \mathcal{H}_{S}^{(m)} .
\end{gathered}
$$

Again, $\left\{p_{i}\right\}$ is arbitrary probability distribution and $\rho_{S}^{(i)}$ is arbitrary state on $\mathcal{H}_{S}^{(i)}$, but $\tilde{\omega}_{S E}^{(i)}$ is a fixed state on $\mathcal{H}_{S}^{(i)} \otimes \mathcal{H}_{E}$ and $\tilde{\omega}_{i}$ are fixed states on $\mathcal{H}_{E}$. The operator sum representations for the $\mathrm{CP}$ reduced dynamics, given by the initial states $\rho_{S E}$ in Eqs. (9) and (10), are given in Ref. [4].

The final generalization (prior to our present work) is that of Ref. [6], which we will write it in the form introduced in Ref. [7]. There, it has been shown that if the set of initial states $\rho_{S E}$ is given by

$$
\begin{gathered}
\mathcal{S}=\left\{\rho_{S E}=\bigoplus_{i} p_{i} \rho_{L_{i}} \otimes \tilde{\omega}_{R_{i} E}\right\}, \\
\mathcal{H}_{S}=\bigoplus_{i} \mathcal{H}_{L_{i}} \otimes \mathcal{H}_{R_{i}},
\end{gathered}
$$

then the reduced dynamics of the system, given by Eq. (2) with arbitrary $U$, is a CP map. In the above equation, $\left\{p_{i}\right\}$ is arbitrary probability distribution, $\rho_{L_{i}}$ is arbitrary state on $\mathcal{H}_{L_{i}}$, but $\tilde{\omega}_{R_{i} E}$ is a fixed state on $\mathcal{H}_{R_{i}} \otimes \mathcal{H}_{E}$.

It can be shown simply that all the previous sets, given in Eqs. (5), (7), (9) and (10), are special cases of Eq. (11) [7]. For example, the factorized initial state $\rho_{S E}=\rho_{S} \otimes \tilde{\omega}_{E}$ is due to the case that the summation in Eq. (11) includes only one term; so $\mathcal{H}_{S}=\mathcal{H}_{L} \otimes \mathcal{H}_{R}$, where $\mathcal{H}_{R}$ is a trivial one-dimensional Hilbert space. In addition, the operator sum representation for the CP reduced dynamics of the system, with the initial $\rho_{S E} \in \mathcal{S}$ in Eq. (11) and arbitrary $U$ in Eq.(2), is given in Ref. [7].
Now an important result, proven in Ref. [7], is that Eq. (11) is in fact the final possible generalization, if we restrict ourselves to the case of $\mathrm{CP}$ assignment map. In other words, the set of initial $\rho_{S E}$ is given by Eq. (11), if and only if, the assignment map is a CP map. In the next section, we restate this result, using the framework introduced in Ref. [8], which will help to clarify this result better and allow us to generalize it in Sec. V.

Let us end this section with an additional remark. The set $\mathcal{S}$, in Eq. (11), can be written as the steered set from a fixed tripartite state $\tilde{\omega}_{A S E}$. We define

$$
\tilde{\omega}_{A S E}=\bigoplus_{i} \tilde{q}_{i} \tilde{\omega}_{A L_{i}} \otimes \tilde{\omega}_{R_{i} E},
$$

on the Hilbert space $\mathcal{H}_{A} \otimes \mathcal{H}_{S} \otimes \mathcal{H}_{E}$, where $\mathcal{H}_{A}$ is an ancillary Hilbert space, $\left\{\tilde{q}_{i}\right\}$ is a probability distribution, $\tilde{\omega}_{A L_{i}}$ is a state on $\mathcal{H}_{A} \otimes \mathcal{H}_{L_{i}}$, and $\tilde{\omega}_{R_{i} E}$ are those states introduced in Eq. (11), on $\mathcal{H}_{R_{i}} \otimes \mathcal{H}_{E}$. The set of steered states, from performing measurements on the part $A$ of $\tilde{\omega}_{A S E}$, is $[6,7]$ :

$$
\mathcal{S}=\left\{\rho_{S E}=\frac{\operatorname{Tr}_{\mathrm{A}}\left[\left(P_{A} \otimes I_{S E}\right) \tilde{\omega}_{A S E}\right]}{\operatorname{Tr}\left[\left(P_{A} \otimes I_{S E}\right) \tilde{\omega}_{A S E}\right]}, P_{A}>0\right\}
$$

where $P_{A}$ is arbitrary positive operator on $\mathcal{H}_{A}$ such that $\operatorname{Tr}\left[\left(P_{A} \otimes I_{S E}\right) \tilde{\omega}_{A S E}\right]>0$ and $I_{S E}$ is the identity operator on $\mathcal{H}_{S} \otimes \mathcal{H}_{E}$. Note that, up to a positive factor, $P_{A}$ can be considered as an element of a POVM. Now, by an appropriate choice of the states $\tilde{\omega}_{A L_{i}}$ in Eq. (12), it can be shown that the steered set $\mathcal{S}$ in Eq. (13) coincides with that of Eq. (11) [7].

A tripartite state which can be decomposed as Eq. (12) is called a Markov state [16]. Therefore, for the steered set from a Markov state $\tilde{\omega}_{A S E}$, the reduced dynamics of the system, for arbitrary $U$ in Eq. (2), is CP. Interestingly, the reverse is also true: If for a steered set, from a fixed tripartite state $\tilde{\omega}_{A S E}$, the reduced dynamics of the system, for arbitrary $U$, is CP, then $\tilde{\omega}_{A S E}$ is a Markov state [6].

A Markov state $\tilde{\omega}_{A S E}$ possesses another interesting property too: For arbitrary unitary evolution of the system-environment $U_{S E}, \tilde{\omega}_{A S E}^{\prime}=i d_{A} \otimes A d_{U_{S E}}\left(\tilde{\omega}_{A S E}\right)$ where $i d_{A}$ is the identity map on $A$, the following quantum data processing inequality is satisfied [6]:

$$
I(A: S)_{\tilde{\omega}_{A S}} \geq I(A: S)_{\tilde{\omega}_{A S}^{\prime}},
$$

where $\tilde{\omega}_{A S}=\operatorname{Tr}_{E}\left(\tilde{\omega}_{A S E}\right)$ and $\tilde{\omega}_{A S}^{\prime}=\operatorname{Tr}_{E}\left(\tilde{\omega}_{A S E}^{\prime}\right)$. In the above equation, $I(A: S)$ is the mutual information. For a bipartite state $\omega_{A S}$, the mutual information is defined as $I(A: S)_{\omega}=S\left(\omega_{A}\right)+S\left(\omega_{S}\right)-S\left(\omega_{A S}\right)$, where $\omega_{A}=\operatorname{Tr}_{S}\left(\omega_{A S}\right)$ and $\omega_{S}=\operatorname{Tr}_{\mathrm{A}}\left(\omega_{A S}\right)$ and $S(\omega)$ is the von Neumann entropy of the state $\omega: S(\omega)=-\operatorname{Tr}(\omega \log \omega)$ [1]. Interestingly, the reverse is also true: If, for a tripartite state $\tilde{\omega}_{A S E}$, the inequality (14) is satisfied for arbitrary $U_{S E}$, then $\tilde{\omega}_{A S E}$ is a Markov state as Eq. (12) [6].

In summary, we have seen that for the steered set $\mathcal{S}$, as Eq. (13), the reduced dynamics of the system is CP, 
for arbitrary $U_{S E}$, if and only if, the $\tilde{\omega}_{A S E}$ is a Markov state. On the other hand, the $\tilde{\omega}_{A S E}$ is a Markov state, if and only if, the quantum data processing inequality (14) is satisfied for arbitrary $U_{S E}$. Therefore, for the steered set $\mathcal{S}$ in Eq. (13), the CP-ness of the reduced dynamics of the system, for arbitrary $U_{S E}$, is equivalent to the satisfaction of the quantum data processing inequality (14), for arbitrary $U_{S E}[6]$.

\section{WHEN THE ASSIGNMENT MAP IS CP}

As mentioned in Sec. II, if the reduced dynamics of the system in Eq. (2) can be represented by a linear map $\Psi$, then it can be shown simply that this $\Psi$ is Hermitian. Consider the set $\mathcal{S}_{S}$ of initial $\rho_{S}=\operatorname{Tr}_{\mathrm{E}}\left(\rho_{S E}\right)$ for which $\rho_{S}^{\prime}=\operatorname{Tr}_{\mathrm{E}} \circ A d_{U}\left(\rho_{S E}\right)$ are given by $\Psi\left(\rho_{S}\right)$ : $\rho_{S}^{\prime}=\Psi\left(\rho_{S}\right) . \quad \mathcal{S}_{S}$ is called the physical domain [8] or the compatibility domain [13] of $\Psi$. Since the Hilbert space of the system $\mathcal{H}_{S}$ is finite-dimensional, one can find a set $\mathcal{S}_{S}^{\prime} \subset \mathcal{S}_{S}$ including a finite number of $\rho_{S}^{(i)} \in$ $\mathcal{S}_{S}$ which are linearly independent and other states in $\mathcal{S}_{S}$ can be decomposed as linear combinations of them: $\mathcal{S}_{S}^{\prime}=\left\{\rho_{S}^{(1)}, \rho_{S}^{(2)}, \cdots, \rho_{S}^{(m)}\right\}$, where $m$ is an integer and $m \leq\left(d_{S}\right)^{2}$ ( $d_{S}$ is the dimension of $\mathcal{H}_{S}$, so $\left(d_{S}\right)^{2}$ is the dimension of $\mathcal{L}\left(\mathcal{H}_{S}\right)$, the space of linear operators on $\left.\mathcal{H}_{S}\right)$, and, for each $\rho_{S} \in \mathcal{S}_{S}$, we have $\rho_{S}=\sum_{i=1}^{m} a_{i} \rho_{S}^{(i)}$ with real $a_{i}$. Now any Hermitian operator $A$, which can be expanded by $\rho_{S}^{(i)} \in \mathcal{S}_{S}^{\prime}$, is obviously mapped by the linear map $\Psi$ to a Hermitian operator: $A=\sum_{i=1}^{m} c_{i} \rho_{S}^{(i)}$, with real $c_{i}$, so $\Psi(A)=\sum_{i=1}^{m} c_{i} \Psi\left(\rho_{S}^{(i)}\right) . \Psi\left(\rho_{S}^{(i)}\right)$ are density operators, therefore $\Psi(A)$ is Hermitian. In other words, $\Psi(A)$ is Hermitian for all Hermitian operators $A$ which can be expanded by $\rho_{S}^{(i)} \in \mathcal{S}_{S}^{\prime}$. Even if $m<\left(d_{S}\right)^{2}$ one can easily extend $\Psi$ to construct a linear Hermitian map on the whole $\mathcal{L}\left(\mathcal{H}_{S}\right)$.

A general framework for linear trace-preserving Hermitian maps, arising from Eq. (2), has been developed in Ref. [8]. This framework can be used to prove interesting results. For example, in Ref. [17], it has been shown that the physically relevant part of any Hermitian map $\Psi$, can represent a possible reduced dynamics of the system. By physically relevant part of $\Psi$, we mean the restriction of $\Psi$ to those initial states $\rho_{S}$ which are mapped by $\Psi$ to density operators, i.e. $\Psi\left(\rho_{S}\right)$ are also density operators. In the following, we use this framework to restate the main result of Ref. [7].

For the finite-dimensional Hilbert space $\mathcal{H}_{S} \otimes \mathcal{H}_{E}$, the set of linear operators on $\mathcal{H}_{S} \otimes \mathcal{H}_{E}$, i.e. $\mathcal{L}\left(\mathcal{H}_{S} \otimes \mathcal{H}_{E}\right)$, is also a finite-dimensional vector space. Consider the set $\mathcal{S} \subseteq \mathcal{D}_{S E}$, where $\mathcal{D}_{S E}$ is the set of density operators on $\mathcal{H}_{S} \otimes \mathcal{H}_{E}$. Obviously $\mathcal{S} \subset \mathcal{L}\left(\mathcal{H}_{S} \otimes \mathcal{H}_{E}\right)$; so one can find a set $\mathcal{S}^{\prime}$, including a finite number of $\rho_{S E}^{(l)}$ in $\mathcal{S}$, which are linearly independent and decompose other elements of $\mathcal{S}$ as linear combinations of these $\rho_{S E}^{(l)} \in \mathcal{S}^{\prime}$. For example, if $\mathcal{S}^{\prime}=\left\{\rho_{S E}^{(1)}, \rho_{S E}^{(2)}, \cdots, \rho_{S E}^{(n)}\right\}$, we can write each member of $\mathcal{S}$ as $\rho_{S E}=\sum_{l=1}^{n} a_{l} \rho_{S E}^{(l)}$, where the real coefficients $a_{l}$ are unique. Finally, let us define $\mathcal{V} \subseteq \mathcal{L}\left(\mathcal{H}_{S} \otimes \mathcal{H}_{E}\right)$ as the subspace spanned by $\rho_{S E}^{(l)} \in \mathcal{S}^{\prime}$; i.e., for each $X \in \mathcal{V}$, we have $X=\sum_{l} c_{l} \rho_{S E}^{(l)}$ with unique complex coefficients $c_{l}$. Obviously $\mathcal{S} \subset \mathcal{V}$.

Now consider a linear map $\Psi$ on $\operatorname{Tr}_{E} \mathcal{V}$ in the form of Eq. (2). So, for each $x \in \operatorname{Tr}_{E} \mathcal{V}$, we have $\left(x=\operatorname{Tr}_{E} X\right.$, $X \in \mathcal{V}):$

$$
x^{\prime}=\Psi(x)=\operatorname{Tr}_{\mathrm{E}} \circ A d_{U}(X)=\operatorname{Tr}_{\mathrm{E}}\left(U X U^{\dagger}\right) .
$$

The first obvious requirement that such a map $\Psi$ can be defined, is the $U$-consistency of the $\mathcal{V}$ [8]; i.e. if for two operators $X_{1}$ and $X_{2} \in \mathcal{V}$, we have $\operatorname{Tr}_{E} X_{1}=\operatorname{Tr}_{E} X_{2}=x$, then we must have $\operatorname{Tr}_{E} \circ A d_{U}\left(X_{1}\right)=\operatorname{Tr}_{E} \circ A d_{U}\left(X_{2}\right)=$ $\Psi(x)$. In Ref. [8], it has been shown that if $\mathcal{S}$ is convex and $U$-consistent, then $\mathcal{V}$ is $U$-consistent too. Now, one can define an assignment map $\Lambda_{1}\left(\Lambda_{2}\right)$ in the following form: $\Lambda_{1}(x)=X_{1}\left(\Lambda_{2}(x)=X_{2}\right)$. So $\Psi$ in Eq. (15) can be written as $\Psi(x)=\operatorname{Tr}_{E} \circ A d_{U} \circ \Lambda_{1}(x)(\Psi(x)=$ $\left.\operatorname{Tr}_{E} \circ A d_{U} \circ \Lambda_{2}(x)\right)$.

It has been shown in Ref. [8] that if $\mathcal{V}$ is $U$-consistent for arbitrary unitary $U \in \mathcal{L}\left(\mathcal{H}_{S} \otimes \mathcal{H}_{E}\right)$, then for each $x \in$ $\operatorname{Tr}_{E} \mathcal{V}$, there is only one $X \in \mathcal{V}$ for which we have $\operatorname{Tr}_{E} X=$ $x$. So, there is only one way to define the assignment map $\Lambda$.

The assignment map $\Lambda$ is Hermitian and so has an operator sum representation as Eq. (3) [8]. Now let's restrict ourselves to the case that $\Lambda$ is in addition a $\mathrm{CP}$ map and so has an operator sum representation as Eq. (4). (The extension of) $\Lambda$ is a map form $\mathcal{L}\left(\mathcal{H}_{S}\right)$ to $\mathcal{L}\left(\mathcal{H}_{S} \otimes\right.$ $\left.\mathcal{H}_{E}\right)$. To make the input and output spaces the same, we redefine $\Lambda$ in the following way: If for a $x \in \mathcal{L}\left(\mathcal{H}_{S}\right)$ we have $\Lambda(x)=X \in \mathcal{L}\left(\mathcal{H}_{S} \otimes \mathcal{H}_{E}\right)$, we set $\Lambda\left(x \otimes\left|0_{E}\right\rangle\left\langle 0_{E}\right|\right)=$ $X$ where $\left|0_{E}\right\rangle$ is a fixed state in $\mathcal{H}_{E}$. This redefinition helps us to write $\Lambda$ in the following form. One can find an ancillary Hilbert space $\mathcal{H}_{C}$, a fixed state $\left|0_{C}\right\rangle \in \mathcal{H}_{C}$ and a unitary operator $V$ on $\mathcal{H}_{S} \otimes \mathcal{H}_{E} \otimes \mathcal{H}_{C}$ in such a way that the CP map $\Lambda$ can be written as [1]:

$$
\begin{aligned}
& \Lambda(x)=\Lambda\left(x \otimes\left|0_{E}\right\rangle\left\langle 0_{E}\right|\right) \\
& =\operatorname{Tr}_{C}\left(V\left(x \otimes\left|0_{E}\right\rangle\left\langle 0_{E}|\otimes| 0_{C}\right\rangle\left\langle 0_{C}\right|\right) V^{\dagger}\right) .
\end{aligned}
$$

The next observation is based on the useful result of Ref. [18]. Note that for arbitrary state $\rho_{S} \in \operatorname{Tr}_{\mathrm{E}} \mathcal{V}$, we have

$$
\hat{\Phi}\left(\rho_{S}\right) \equiv \operatorname{Tr}_{\mathrm{E}} \circ \Lambda\left(\rho_{S}\right)=\rho_{S}
$$

$\hat{\Phi}$ is a $\mathrm{CP}$ map, since $\Lambda$ is a CP map by assumption and the partial trace $\operatorname{Tr}_{\mathrm{E}}$ is also a CP map [1]. In addition note that

$$
\hat{\Phi}\left(\rho_{S}\right)=\operatorname{Tr}_{\mathrm{EC}}\left(V\left(\rho_{S} \otimes\left|0_{E}\right\rangle\left\langle 0_{E}|\otimes| 0_{C}\right\rangle\left\langle 0_{C}\right|\right) V^{\dagger}\right),
$$

with the unitary $V$ introduced in Eq. (16).

In Ref. [18], it has been shown that if for a set of states $\mathcal{S}_{S} \equiv \operatorname{Tr}_{\mathrm{E}}(\mathcal{V}) \cap \mathcal{D}_{S}$ (where $\mathcal{D}_{S}$ is the set of density 
operators on $\left.\mathcal{H}_{S}\right)$ and a CP map $\hat{\Phi}$, we have $\hat{\Phi}\left(\rho_{S}\right)=\rho_{S}$ for all $\rho_{S} \in \mathcal{S}_{S}$, then there exists a decomposition of the Hilbert space $\mathcal{H}_{S}$ as $\mathcal{H}_{S}=\bigoplus_{i} \mathcal{H}_{L_{i}} \otimes \mathcal{H}_{R_{i}}$ such that:

(1) each $\rho_{S} \in \mathcal{S}_{S}$ can be decomposed as

$$
\rho_{S}=\bigoplus_{i} p_{i} \rho_{L_{i}} \otimes \tilde{\omega}_{R_{i}}
$$

where the probability distribution $\left\{p_{i}\right\}$ and states $\rho_{L_{i}} \in$ $\mathcal{D}_{L_{i}}$ are dependent on $\rho_{S}$, but the states $\tilde{\omega}_{R_{i}} \in \mathcal{D}_{R_{i}}$ are fixed for all $\rho_{S}$; and

(2) the unitary $V$ in Eq. (18) is in the form of

$$
V=\bigoplus_{i} I_{L_{i}} \otimes V_{R_{i} E C},
$$

where $I_{L_{i}}$ is the identity operator on $\mathcal{H}_{L_{i}}$ and $V_{R_{i} E C}$ is a unitary operator on $\mathcal{H}_{R_{i}} \otimes \mathcal{H}_{E} \otimes \mathcal{H}_{C}$.

Combining Eqs. (16) and (20) gives us:

$$
\Lambda=\bigoplus_{i} i d_{L_{i}} \otimes \Lambda_{R_{i}}
$$

where $i d_{L_{i}}$ is the identity map on $\mathcal{L}\left(\mathcal{H}_{L_{i}}\right)$ and $\Lambda_{R_{i}}$ : $\mathcal{L}\left(\mathcal{H}_{R_{i}}\right) \rightarrow \mathcal{L}\left(\mathcal{H}_{R_{i}} \otimes \mathcal{H}_{E}\right)$ is a $\mathrm{CP}$ assignment map on $\mathcal{L}\left(\mathcal{H}_{R_{i}}\right)$. Using Eqs. (19) and (21), we have:

$$
\Lambda\left(\rho_{S}\right)=\bigoplus_{i} p_{i} \rho_{L_{i}} \otimes \Lambda_{R_{i}}\left(\tilde{\omega}_{R_{i}}\right)
$$

for each $\rho_{S} \in \mathcal{S}_{S} . \Lambda_{R_{i}}$ is a $\mathrm{CP}$ map (on $\mathcal{L}\left(\mathcal{H}_{R_{i}}\right)$ ), so it maps the state $\tilde{\omega}_{R_{i}}$ to a state $\tilde{\omega}_{R_{i} E} \in \mathcal{D}_{R_{i} E}$. Therefore:

$$
\rho_{S E}=\Lambda\left(\rho_{S}\right)=\bigoplus_{i} p_{i} \rho_{L_{i}} \otimes \tilde{\omega}_{R_{i} E},
$$

which is in the form of Eq. (11). Note that $\tilde{\omega}_{R_{i}}$ are fixed for all $\rho_{S} \in \mathcal{S}_{S}$, and so are $\tilde{\omega}_{R_{i} E}$.

In summary, we have seen that if:

(1) $\mathcal{V}$ is a $U$-consistent subspace of $\mathcal{L}\left(\mathcal{H}_{S} \otimes \mathcal{H}_{E}\right)$ for all $U$, and

(2) (the extension of) the assignment map $\Lambda$ : $\mathcal{L}\left(\mathcal{H}_{S}\right) \rightarrow \mathcal{L}\left(\mathcal{H}_{S} \otimes \mathcal{H}_{E}\right)$ is $\mathrm{CP}$,

then each $\rho_{S} \in \mathcal{S}_{S}=\operatorname{Tr}_{\mathrm{E}}(\mathcal{V}) \cap \mathcal{D}_{S}$ is in the form of Eq. (19), and is mapped by the assignment map $\Lambda$ to a $\rho_{S E} \in \mathcal{S} \equiv \mathcal{V} \cap \mathcal{D}_{S E}$ which is given by Eq. (22). Also note that assuming that the assignment map $\Lambda$ is CP results in the complete positivity of the reduced dynamical $\operatorname{map} \Psi=\operatorname{Tr}_{\mathrm{E}} \circ A d_{U} \circ \Lambda$.

In other words, the complete positivity of the assignment map $\Lambda$ assures that the set of initial states $\rho_{S E}$ (leading to CP reduced dynamics) is given by Eq. (11) [7]. Reversely, if the set of initial states $\rho_{S E}$ is given by $\mathcal{S}$ in Eq. (11), then it can be shown simply, by explicit construction of $\Lambda$ [7], that the assignment map $\Lambda$ is $\mathrm{CP}$.

Let's end this section with the following point. In the previous section, we have seen that the set $\mathcal{S}$ in Eq. (11) can be written as the steered set, i.e. as Eq. (13), from the fixed Markov state $\tilde{\omega}_{A S E}$, in Eq. (12). For the $U$ consistency of a steered set $\mathcal{S}$, for arbitrary $U \in \mathcal{L}\left(\mathcal{H}_{S} \otimes\right.$
$\mathcal{H}_{E}$ ), a one-to-one correspondence between the members of $\mathcal{S}$ and $\mathcal{S}_{S}=\operatorname{Tr}_{\mathrm{E}}(\mathcal{S})$ is required. For a Markov state $\tilde{\omega}_{A S E}$, the existence of this one-to-one correspondence is proven in Ref. [19].

\section{WHEN $\mathcal{V}$ IS $U$-CONSISTENT FOR A RESTRICTED SET OF UNITARY OPERATORS $U$}

In the previous section, we have restated the main result of Ref. [7]. We saw that the two conditions of $U$ consistency for arbitrary $U$ and the CP-ness of the assignment map, lead to Eq. (11). When the set of initial states $\rho_{S E}$ is given by $\mathcal{S}$ in Eq. (11), then the reduced dynamics, for arbitrary $U$, is $\mathrm{CP}$.

What if we relax the condition of $U$-consistency for arbitrary $U$ ? As we will see in the following, this relaxation leads to a generalization of Eq. (11); i.e. we will find a set $\mathcal{S}$ of initial $\rho_{S E}$ which includes the set given in Eq. (11) as a subset and also leads to CP reduced dynamics (for a restricted set of unitary operators $U$ ).

If $\mathcal{V}$ is $U$-consistent for all $U$ in a set $\mathcal{G} \subseteq \mathcal{U}\left(\mathcal{H}_{S} \otimes\right.$ $\left.\mathcal{H}_{E}\right)$, then $\mathcal{V}$ is called $\mathcal{G}$-consistent $\left(\mathcal{U}\left(\mathcal{H}_{S} \otimes \mathcal{H}_{E}\right)\right.$, is the set of all unitary $\left.U \in \mathcal{L}\left(\mathcal{H}_{S} \otimes \mathcal{H}_{E}\right)\right)$. In the previous section, we have seen that if $\mathcal{G}=\mathcal{U}\left(\mathcal{H}_{S} \otimes \mathcal{H}_{E}\right)$, then there is a one-to-one correspondence between the members of $\operatorname{Tr}_{E}(\mathcal{V})$ and $\mathcal{V}$. But if $\mathcal{G}$ is a proper subset of $\mathcal{U}\left(\mathcal{H}_{S} \otimes\right.$ $\mathcal{H}_{E}$ ), then there is no guarantee for such a one-to-one correspondence. Therefore, one may find two different $X_{1}$ and $X_{2} \in \mathcal{V}$ for which we have $\operatorname{Tr}_{E} X_{1}=\operatorname{Tr}_{E} X_{2}=x$. So, for $Y=X_{1}-X_{2}$ we have $\operatorname{Tr}_{E} Y=0$. In other words, there is a subset $\mathcal{V}_{0} \subset \mathcal{V}$ such that for each $Y \in \mathcal{V}_{0}$ we have $\operatorname{Tr}_{E} Y=0$.

Therefore, there is more than one way to define the assignment map. We write:

$$
\tilde{\Lambda}=\Lambda+\mathcal{V}_{0},
$$

i.e. if $\Lambda$ is an assignment map from $\operatorname{Tr}_{E}(\mathcal{V})$ to $\mathcal{V}$ such that $\Lambda(x)=X\left(x \in \operatorname{Tr}_{E}(\mathcal{V})\right.$ and $\left.X \in \mathcal{V}\right)$, then adding $Y \in \mathcal{V}_{0}$ gives us another possible assignment map $\tilde{\Lambda}$.

In general, the assignment map $\Lambda$ is Hermitian [8]. But, in this paper, we restrict ourselves to the case that the assignment map $\Lambda$ is, in addition, CP; i.e. $\Lambda$ in Eq. (23) is a CP map as Eq. (4). Therefore, for this part of the general assignment map $\tilde{\Lambda}$ in Eq. (23), a similar line of reasoning, as appeared in the previous section, can be applied. So, for $\Lambda$ in Eq. (23), we can write Eq. (16) and then Eq. (17) and finally, using the result of Ref. [18], achieve Eq. (22).

Using Eqs. (22) and (23), for each $\rho_{S} \in \mathcal{S}_{S}=\operatorname{Tr}_{\mathrm{E}}(\mathcal{V}) \cap$ $\mathcal{D}_{S}$, we have

$$
\begin{aligned}
\tilde{\Lambda}\left(\rho_{S}\right) & =\Lambda\left(\rho_{S}\right)+\mathcal{V}_{0} \\
= & \bigoplus_{i} p_{i} \rho_{L_{i}} \otimes \tilde{\omega}_{R_{i} E}+\mathcal{V}_{0},
\end{aligned}
$$

where, as before, the probability distribution $\left\{p_{i}\right\}$ and states $\rho_{L_{i}} \in \mathcal{D}_{L_{i}}$ are dependent on $\rho_{S}$, but the states $\tilde{\omega}_{R_{i} E} \in \mathcal{D}_{R_{i} E}$ are fixed for all $\rho_{S} \in \mathcal{S}_{S}$. 
Therefore, for the set

$$
\mathcal{S} \equiv \mathcal{V} \cap \mathcal{D}_{S E}=\left\{\left(\bigoplus_{i} p_{i} \rho_{L_{i}} \otimes \tilde{\omega}_{R_{i} E}+\mathcal{V}_{0}\right) \cap \mathcal{D}_{S E}\right\}
$$

the reduced dynamical map $\Psi=\operatorname{Tr}_{\mathrm{E}} \circ A d_{U} \circ \tilde{\Lambda}$ is $\mathrm{CP}$, since the assignment map $\Lambda$ is CP. Obviously, Eq. (11) is a special case of Eq. (25), due to the case that $\mathcal{V}_{0}$ is null, which is for $\mathcal{G}=\mathcal{U}\left(\mathcal{H}_{S} \otimes \mathcal{H}_{E}\right)$.

In summary, we have proved the following theorem:

Theorem 1. If

(1) $\mathcal{V}$ is a $U$-consistent subspace of $\mathcal{L}\left(\mathcal{H}_{S} \otimes \mathcal{H}_{E}\right)$, for all $U \in \mathcal{G}$ where $\mathcal{G} \subseteq \mathcal{U}\left(\mathcal{H}_{S} \otimes \mathcal{H}_{E}\right)$, and

(2) (the extension of) the assignment map $\Lambda$ : $\mathcal{L}\left(\mathcal{H}_{S}\right) \rightarrow \mathcal{L}\left(\mathcal{H}_{S} \otimes \mathcal{H}_{E}\right)$ is $C P$,

then $\mathcal{S}=\mathcal{V} \cap \mathcal{D}_{S E}$ is given by Eq. (25), which leads to $C P$ reduced dynamical maps $\Psi=\operatorname{Tr}_{\mathrm{E}} \circ A d_{U} \circ \tilde{\Lambda}=\operatorname{Tr}_{\mathrm{E}} \circ A d_{U} \circ \Lambda$ for all $U \in \mathcal{G}$.

Since Eq. (11) is a special case of Eq. (25), the above theorem includes all the previous results in this context as special cases. We end this section with two simple illustrating examples.

Example 1. Consider the case that $\mathcal{S}=\left\{\rho_{S E} \in\right.$ $\left.\mathcal{D}_{S E}: \operatorname{Tr}_{S}\left(\rho_{S E}\right)=\tilde{\omega}_{E}\right\}$, where $\tilde{\omega}_{E} \in \mathcal{D}_{E}$ is a fixed state, and $\mathcal{G}=\left\{U_{s w}\right\}$, where $U_{s w}$ is the swap operator. $\mathcal{S}$ is convex and $\mathcal{G}$-consistent.

Equivalently, we can write $\mathcal{S}$ as

$$
\mathcal{S}=\left\{\left(\rho_{S} \otimes \tilde{\omega}_{E}+\mathcal{V}_{0}\right) \cap \mathcal{D}_{S E}\right\},
$$

where $\rho_{S}$ is an arbitrary state in $\mathcal{D}_{S}$. For this example, the assignment map $\Lambda$ in Eq. (23) is CP, since, for each $x \in \operatorname{Tr}_{E}(\mathcal{V})=\mathcal{L}\left(\mathcal{H}_{S}\right)$, we have $\Lambda(x)=x \otimes \tilde{\omega}_{E}$ which is obviously a CP map.

Therefore, for the set $\mathcal{S}$ in Eq. (26), the reduced dynamical map $\Psi=\operatorname{Tr}_{\mathrm{E}} \circ A d_{U_{s w}} \circ \tilde{\Lambda}$ is $\mathrm{CP}$. The set $\mathcal{S}$ in Eq. (26) obviously includes Eq. (5) as a subset. Recall that Eq. (5) is the set given by Eq. (11) for the case that the summation in Eq. (11) includes only one term and $\mathcal{H}_{R}$ is a trivial one-dimensional Hilbert space.

Example 2. Consider the case that $\mathcal{S}=\mathcal{D}_{S E}$ and $\mathcal{G}=\left\{U_{S} \otimes U_{E}\right\}$, where $U_{S}\left(U_{E}\right)$ are arbitrary unitary operators on $\mathcal{H}_{S}\left(\mathcal{H}_{E}\right)$. $\mathcal{S}$ is convex and $\mathcal{G}$-consistent and we can write it as

$$
\mathcal{S}=\left\{\left(\rho_{S} \otimes \tilde{\omega}_{E}+\mathcal{V}_{0}\right) \cap \mathcal{D}_{S E}\right\},
$$

where $\rho_{S}$ is an arbitrary state in $\mathcal{D}_{S}$ and $\tilde{\omega}_{E}$ is (arbitrary chosen) fixed state in $\mathcal{D}_{E}$. Note that $\mathcal{S}$ in Eq. (27) differs from $\mathcal{S}$ in Eq. (26), because of the difference between $\mathcal{V}_{0}$ in the two mentioned equations. In the current example, $\mathcal{V}=\mathcal{L}\left(\mathcal{H}_{S} \otimes \mathcal{H}_{E}\right)$. So $\mathcal{V}_{0}=\operatorname{ker} \operatorname{Tr}_{\mathrm{E}}$, i.e. the set of all $Y \in \mathcal{L}\left(\mathcal{H}_{S} \otimes \mathcal{H}_{E}\right)$ for which $\operatorname{Tr}_{\mathrm{E}}(Y)=0$.

Writing $\mathcal{S}$ as Eq. (27) shows that the assignment map $\Lambda$ in this example is the same as $\Lambda$ in the previous example. The CP-ness of $\Lambda$ results in the CP-ness of the reduced dynamical maps $\Psi=\operatorname{Tr}_{\mathrm{E}} \circ A d_{U} \circ \tilde{\Lambda}$, for all $U \in \mathcal{G}$.

Obviously, the set $\mathcal{S}=\mathcal{D}_{S E}$ in this example, which yields CP reduced dynamics, is larger than any set which can be constructed by Eq. (11).

Note that this example is, in fact, restating the result of Ref. [12], using the Theorem 1.

It is also worth noting that though the assignment map $\Lambda$, in the above two examples, became the same as the one first introduced by Pechukas [13, 20], but, because in the above examples $\mathcal{G} \neq \mathcal{U}\left(\mathcal{H}_{S} \otimes \mathcal{H}_{E}\right)$ and so $\mathcal{V}_{0}$ is not null, the assignment map $\tilde{\Lambda}$ is not the same as the Pechukas's one.

\section{SUMMARY}

Though there was a tendency to assume the CP maps as the only possible quantum dynamics of a system, we have seen in Sec. II that this is not the case, at least, for open quantum systems.

In fact, the CP-ness of the reduced dynamics of the system, for arbitrary $U$ in Eq. (2), has been proven only for some restricted sets of initial $\rho_{S E}$, which we reviewed them in Sec. III. All the sets given in this section can be written as special cases of Eq. (11).

An important result in this context is that of Ref. [7], where it has been shown that if the assignment map is $\mathrm{CP}$, then the set of initial $\rho_{S E}$ is given by Eq. (11).

In Sec. IV, we restated the mentioned result of Ref. [7], using the framework introduced in Ref. [8]. This treatment highlighted the condition of $U$-consistency for arbitrary $U$, which is needed to achieve the result of Ref. [7]. In Ref. [7], it has been assumed implicitly that there is only one way to define the assignment map, which is true only when the $U$-consistency condition is valid for arbitrary $U$.

Finally, our treatment led us to the main result of this paper, which was given in Sec. V. There, we relaxed the condition of $U$-consistency for arbitrary $U$ and found the most general possible set of initial $\rho_{S E}$ for which the assignment map $\Lambda$ is $\mathrm{CP}$ and so the reduced dynamical maps are also CP (for a restricted set of $U$ in Eq. (2)). This set, which was given in Eq. (25), includes Eq. (11) as a special case. Therefore, it includes all the previously found sets as special cases as well.
[1] M. A. Nielsen and I. L. Chuang, Quantum Computation and Quantum Information (Cambridge University Press, Cambridge, 2000).
[2] C. A. Rodrguez-Rosario, K. Modi, A.-m. Kuah, A. Shaji and E. C. G. Sudarshan, Completely positive maps and classical correlations, J. Phys. A: Math. Theor. 41, 
205301 (2008).

[3] A. Shabani and D. A. Lidar, Vanishing quantum discord is necessary and sufficient for completely positive maps, Phys. Rev. Lett. 102, 100402 (2009); Erratum: Vanishing quantum discord is necessary and sufficient for completely positive maps [Phys. Rev. Lett. 102, 100402 (2009)], Phys. Rev. Lett. 116, 049901 (2016).

[4] L. Liu and D. M. Tong, Completely positive maps within the framework of direct-sum decomposition of state space, Phys. Rev. A 90, 012305 (2014).

[5] A. Brodutch, A. Datta, K. Modi, A. Rivas and C. A. Rodrguez-Rosario, Vanishing quantum discord is not necessary for completely positive maps, Phys. Rev. A 87, 042301 (2013).

[6] F. Buscemi, Complete positivity, Markovianity, and the quantum data-processing inequality, in the presence of initial system-environment correlations, Phys. Rev. Lett. 113, 140502 (2014).

[7] X.-M. Lu, Structure of correlated initial states that guarantee completely positive reduced dynamics, Phys. Rev. A 93, 042332 (2016).

[8] J. M. Dominy, A. Shabani and D. A. Lidar, A general framework for complete positivity, Quantum Inf. Process. 15, 465 (2016).

[9] P. Stelmachovic and V. Buzek, Dynamics of open quantum systems initially entangled with environment: Beyond the Kraus representation, Phys. Rev. A 64, 062106 (2001); Erratum: Dynamics of open quantum systems initially entangled with environment: Beyond the Kraus representation [Phys. Rev. A 64, 062106 (2001)], Phys. Rev. A 67, 029902(E)(2003).
[10] K. M. F. Romero, P. Talkner and P. Hanggi, Is the dynamics of open quantum systems always linear?, Phys. Rev. A 69, 052109 (2004).

[11] H. A. Carteret, D. R. Terno and K. Zyczkowski, Dynamics beyond completely positive maps: Some properties and applications, Phys. Rev. A 77, 042113 (2008).

[12] D. Salgado and J. L. Sanchez-Gomez, Comment on "Dynamics of open quantum systems initially entangled with environment: Beyond the Kraus representation" [PRA 64, 062106 (2001)], arXiv:quant-ph/0211164 (2002).

[13] T. F. Jordan, A. Shaji and E. C. G. Sudarshan, Dynamics of initially entangled open quantum systems, Phys. Rev. A 70, 052110 (2004).

[14] E. C. G. Sudarshan, P. M. Mathews and J. Rau, Stochastic dynamics of quantum-mechanical systems, Phys. Rev. 121, 920 (1961).

[15] B. Vacchini and G. Amato, Reduced dynamical maps in the presence of initial correlations, Sci. Rep. 6, 37328 (2016).

[16] P. Hayden, R. Jozsa, D. Petz and A. Winter, Structure of states which satisfy strong subadditivity of quantum entropy with equality, Commun. Math. Phys. 246, 359 (2004).

[17] J. M. Dominy and D. A. Lidar, Beyond complete positivity, Quantum Inf. Process. 15, 1349 (2016).

[18] M. Koashi and N. Imoto, Operations that do not disturb partially known quantum states, Phys. Rev. A 66, 022318 (2002).

[19] I. Sargolzahi, Reference state for arbitrary U-consistent subspace, J. Phys. A: Math. Theor. 51, 315301 (2018).

[20] P. Pechukas, Reduced dynamics need not be completely positive, Phys. Rev. Lett. 73, 1060 (1994). 